\title{
Magnetosheath excursion and the relevant transport process at the magnetopause
}

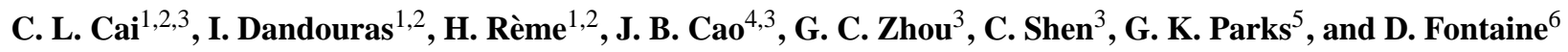 \\ ${ }^{1}$ University of Toulouse, UPS, CESR, 9 avenue colonel Roche, 31028 Toulouse cedex 4, France \\ ${ }^{2}$ CNRS, UMR5187, 31028 Toulouse, France \\ ${ }^{3}$ State Key Laboratory of Space Weather, Center for Space Science and Applied Research, 100080 Beijing, China \\ ${ }^{4}$ School of Astronautics, Beijing University of Aeronautics and Astronautics, 100191 Beijing, China \\ ${ }^{5}$ Space Sciences Laboratory, University of California, Berkeley, CA 94720, USA \\ ${ }^{6}$ Centre d'Etudes des Environnements Terrestre et Planétaires, Centre National de la Recherche Scientifique, \\ 78140 Vélizy, France
}

Received: 7 May 2009 - Accepted: 26 June 2009 - Published: 3 August 2009

\begin{abstract}
A large-amplitude excursion of the magnetosheath (MS) in quiet solar wind conditions on 17 March 2004 was recorded simultaneously by the Cluster and TC-1 spacecraft. During this period, the IMF $B_{z}$ was entirely northward. The coherence between the bow shock motion and magnetopause (MP) motion is revealed and the excursion velocities of the bow shock motion are analyzed. In addition, the relevant plasma transport phenomenon in the form of flux fluctuations below the ion gyrofrequency at the MP is exposed and is interpreted as manifestation of the drift instability. Correlated observations on charge accumulation and electrostatic potential perturbation are recorded by electron measurements in high energy regime, and also the eventual cross-field vortex motion in the nonlinear stage and the consequential mass exchange are exhibited. The present investigation gives some new insight into the MS plasma transport mechanism across the subsolar MP region in quiet solar wind conditions during a period of northward IMF.
\end{abstract}

Keywords. Magnetospheric physics (Magnetosheath; Plasma waves and instabilities) - Space plasma physics (Transport processes)

\section{Introduction}

The shape and location of the bow shock and MP are very sensitive to the solar wind conditions, especially the solar wind kinetic pressure and the orientation of the interplanetary

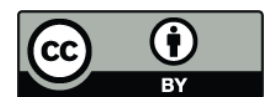

Correspondence to: C. L. Cai

(clcai@cssar.ac.cn) magnetic field (IMF). Observations have indicated that the MP and bow shock are almost always in motion, sometimes at large velocities, even during periods of low solar and geomagnetic activity (Holzer et al., 1966; Anderson et al., 1968). The MP and the bow shock motions are usually found to be coherent over a large distance. Previous statistical studies on the speed of the MP motion showed that typical values were in the range 10-60 km/s (Anderson et al., 1968). Further investigations based on energetic ion remote sensing technique found that the velocities are usually less than $20 \mathrm{~km} / \mathrm{s}$ but for one case, the value was $156 \mathrm{~km} / \mathrm{s}$ (Kaufmann and Konradi, 1969). Time lag measurements from ISEE 1 and 2 found the speeds were in the range $5-380 \mathrm{~km} / \mathrm{s}$, with $80 \%$ falling in the range $10-80 \mathrm{~km} / \mathrm{s}$ (Berchem and Russell, 1982). Recent statistical study of the bow shock motion based on Cluster data shows that typical shock velocities are $35 \mathrm{~km} / \mathrm{s}$, and the fastest one was nearly $150 \mathrm{~km} / \mathrm{s}$ (Horbury et al., 2002).

Although the MP is commonly supposed to be an impenetrable boundary, the well-established existence of plasma of solar wind origin inside the magnetosphere implies that solar wind plasma is able to cross the MP (Phan et al., 2005). A variety of processes has been proposed to account for this transport phenomenon. In the case of southward IMF, it is widely believed that magnetic reconnection is responsible for the transfer of solar wind mass into the magnetosphere. In contrast, during northward IMF which mechanism facilitates the MS plasma to enter into the magnetosphere, especially through the subsolar point region, is still unclear. A possible candidate is the diffusive wave-particle transport by the lower hybrid drift instability (Gary and Eastman, 1979; LaBelle and Treumann, 1988; Treumann et al. 1991). An alternative mechanism is direct large-scale intrusion of plasma

Published by Copernicus Publications on behalf of the European Geosciences Union. 


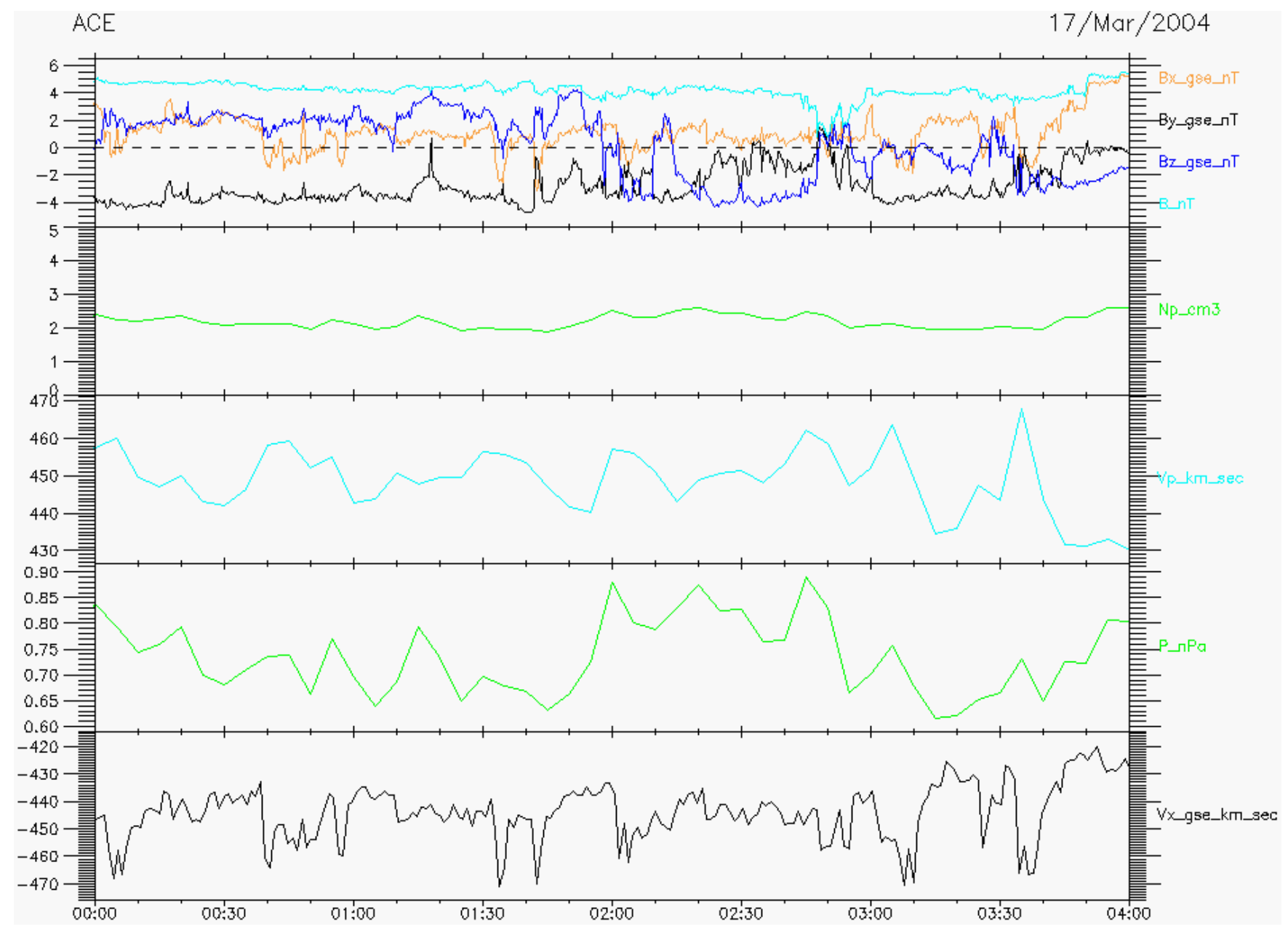

Fig. 1. Solar wind parameters from ACE.

into the magnetosphere by the formation of the mixed transition layer at the MP (Lemaire, 1977; Lemaire and Roth, 1978).

In the present paper, we report a bulk excursion of the MS that was recorded simultaneously by the Cluster and TC1 spacecraft in quiet solar wind conditions during a period of northward IMF on 17 March 2004. The relevant plasma transport phenomenon in the form of flux fluctuations below the ion gyrofrequency at the MP is shown and interpreted as manifestation of the drift instability.

\section{Observations}

Figure 1 shows the solar wind parameters from ACE. During the period 00:00-02:00 UT, the IMF $B_{z}$ was entirely northward, and the total field amplitude was almost constant. The solar wind velocity had only some small fluctuations and the kinetic pressure manifested some modest perturbations. The solar wind condition in this time interval can be considered as moderately quiet. The propagation time for plasma from
L1 to Earth, estimated by the advection shift, is about $55 \mathrm{~min}$. Since the concerned time interval is 02:30-02:50 UT, hence ACE observations before 02:00 UT are relevant. On early 17 March 2004, all the Cluster and TC-1 spacecraft was inbound. The geographical locations are schematically shown in Fig. 2. The four Cluster spacecraft were approximately at $\mathbf{R}=(14.00,-6.87,-9.13)_{\mathrm{GSE}} R_{E}$, and the characteristic scale of the tetrahedron was only about $200 \mathrm{~km}$ at that time. The TC-1 spacecraft was at $\mathbf{R}=(12.03,-4.69,-1.80)_{\mathrm{GSE}} R_{E}$. Figures 3 and 4 show magnetic field measurements and hot ion measurements recorded by Cluster and TC-1 satellites for 02:20-02:55 UT. The magnetic field measurements come from the FGM experiment (Balogh et al., 2001) and the hot ions from HIA sensor of the CIS instrument (Rème et al., 2001). Almost identical HIA instrument is also on board TC1 (Rème et al., 2005). For the 4 Cluster spacecraft, only data from $\mathrm{C} 3$ are shown here(because of the very small size of the tetrahedron all Cluster showed very similar features).

The first panel in Fig. 3 displays the time-energy spectrogram of the low geometrical factor HIA sensor designed 


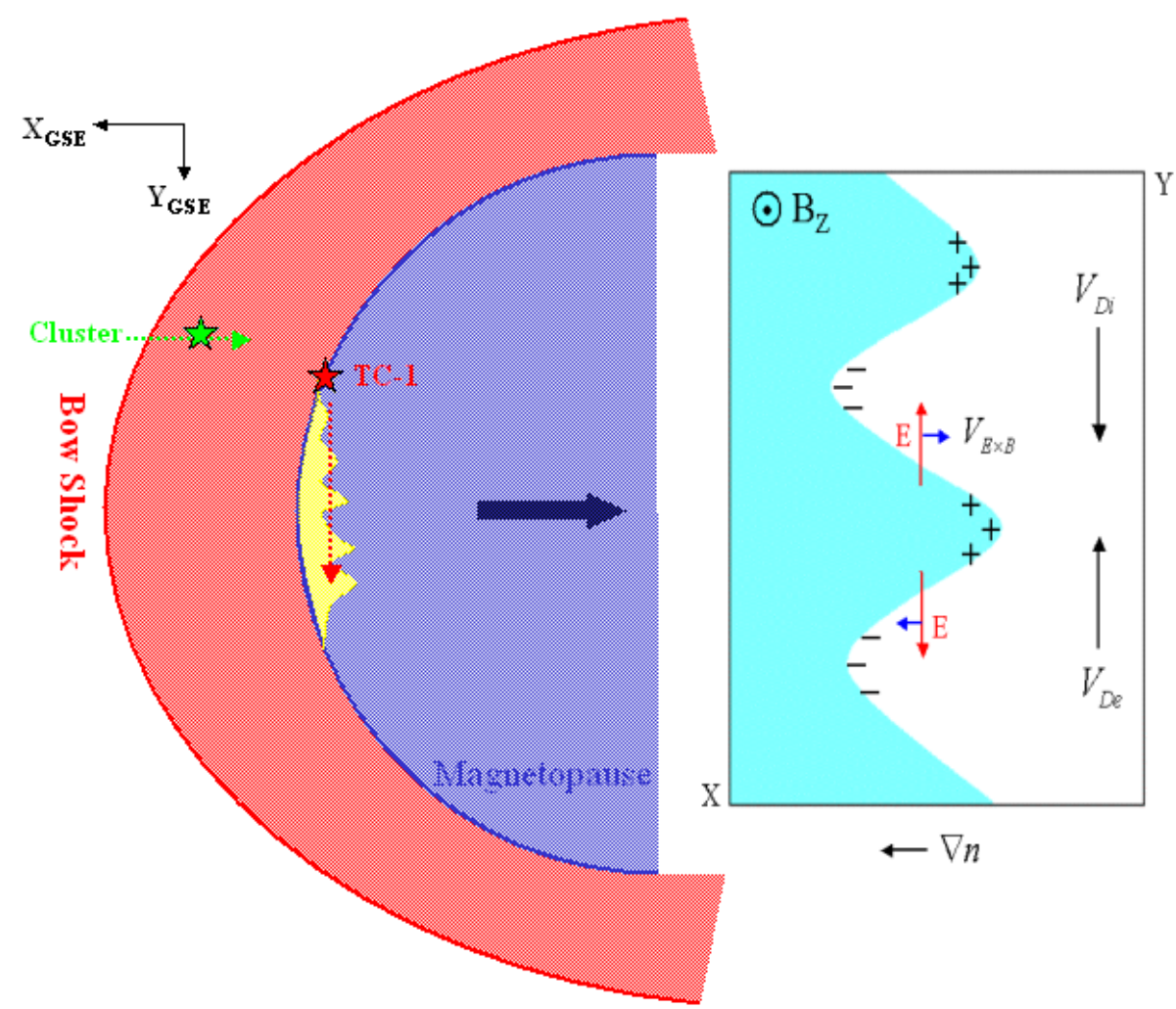

Fig. 2. A schematic of the geographical locations of the Cluster and TC-1 spacecraft projected to the ecliptic plane. The bow shock and the magnetopause are shown by the red and blue bows respectively. The green and red stars and the dashed arrows denote the Cluster and TC-1 spacecraft and their orbits respectively. The ripples at the magnetopause describe the scenario of the drift instability. Meanwhile, its fundamental physical picture is illustrated at the right side. A sinusoidal electrostatic potential perturbation tight ties to a sinusoidal plasma density perturbation, and both them propagate with the plasma diamagnetic drift velocity. For more details see the text.

to detect the solar wind particles. The density and velocities are shown in the second and third panels. The fourth panel shows the time-energy spectrogram of the high geometrical factor C3 HIA sensor, which is designed to detect the MS and magnetospheric particles. The density and velocities from this detector are shown in the fifth and sixth panels. Until 02:30 and after 02:47 UT, the energy spectrum shows almost monoenergetic ions, with velocities nearly in the $\mathrm{x}$-direction (GSE). All these features indicate that the observations were recorded inside the solar wind, while during the interval 02:30-02:47 UT, the observational characteristics indicate that the spacecraft entered into and stayed in the MS. Due to the deceleration and thermalization of the solar wind plasma behind the bow shock, the ion energy spectrum in the MS is spread, and the corresponding density rises to a much higher level. During this period, the bulk velocities also decreased and was deflected in comparison to the solar wind velocities. Likewise, TC-1 was inside the MS till 02:30 and after 02:47 UT as it can be easily identified from Fig. 4 . During the interval 02:30-02:47 UT, it is clear that the observations were recorded in the magnetosphere, where the ion energy spectrum is in a higher energy regime compared to that of the MS. Here, the reason that the density recorded by the low geometrical factor HIA sensor decreases inside the MS is due to the fact that it is in the solar wind mode (instrument effect).

Combining the observations of the Cluster and TC-1 spacecraft, the present event reveals a large-amplitude bulk excursion of the MS. Initially, the Cluster spacecraft was in the solar wind and TC- 1 in the MS. At 02:30 UT, a rapid bulk sunward motion of the MS took place Cluster entered the MS, and TC- 1 encountered the MP. After about $17 \mathrm{~min}$ at 02:47 UT, a rapid earthward motion of the MS occurred. Thus Cluster reentered the solar wind, and TC-1 returned to the MS. An impressive feature of the present event is the good coherence between the bow shock and the MP motion. To expose this point, we added the time-energy spectrogram recorded at the same time by TC- 1 satellite on the bottom panel of Fig. 3. It can be seen that the period of Cluster in the MS exactly coincides with the period of TC- 1 at the MP.

In the present event, the MS magnetic field has components with a positive $B_{z}$, a negative $B_{y}$ and a nearly zero $B_{x}$, while the MP magnetic field is mainly in the $\mathrm{z}$ direction with small $B_{x}$ and $B_{y}$ components. That is, the MS magnetic 

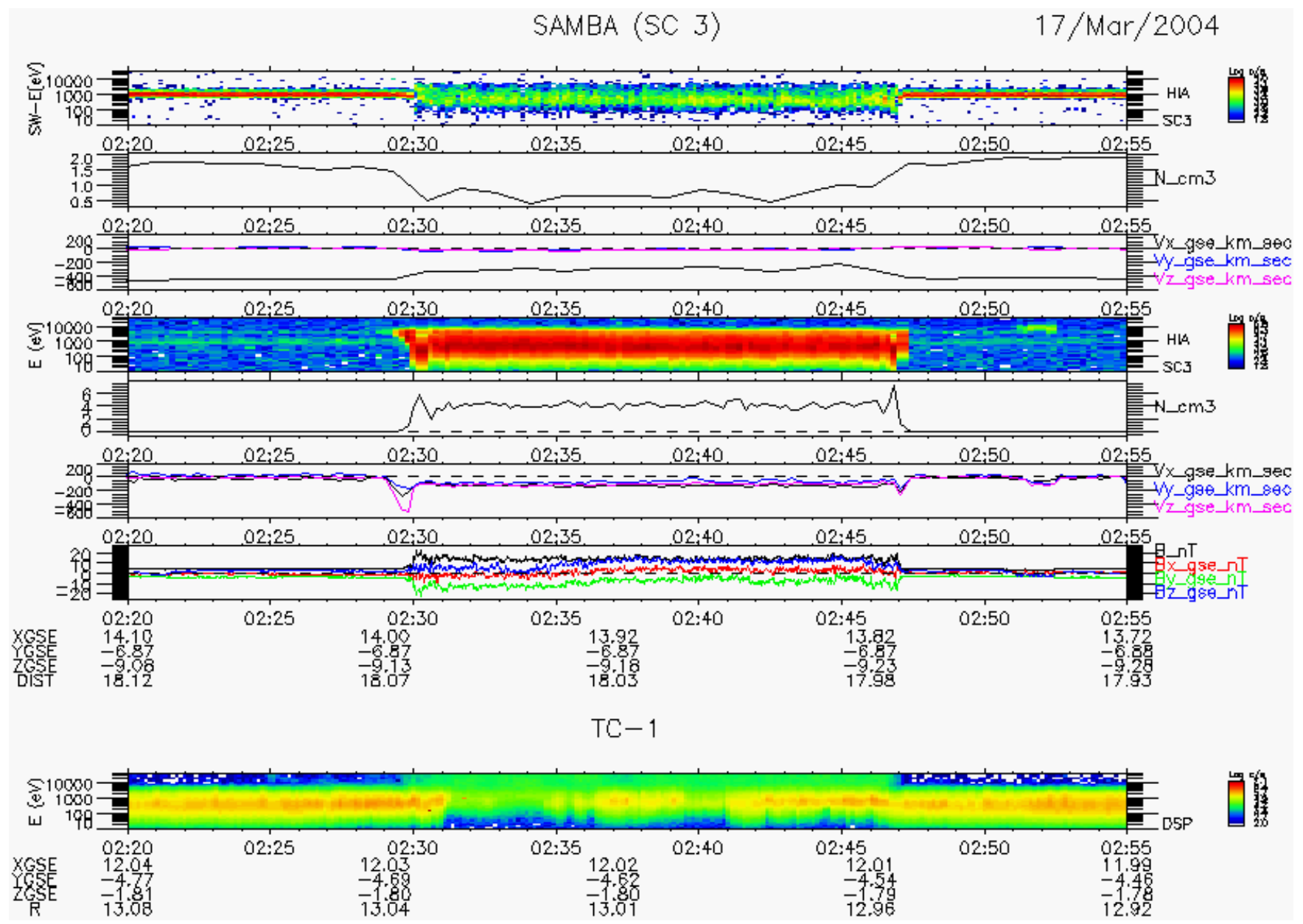

Fig. 3. Magnetic field and hot ion parameters from Cluster C3 . From top, (a-c) time-energy spectrogram and density and velocities of solar wind particles respectively, (d-f) time-energy spectrogram and density and velocities of magnetosheath particles respectively. g) components of magnetic field. However, the last panel is hot ion time-energy spectrogram from TC-1.

field is sheared to the terrestrial magnetic field with an angle about $45^{\circ}$. Based on the temperature of MS ions and the total magnetic field, we can estimate the proton gyroradius to be approximately $80 \mathrm{~km}$ and the gyroperiod about $2 \mathrm{~s}$.

Another remarkable feature recorded by TC- 1 at the MP is the mixture of the MS and magnetospheric ion composition: the high energy ions characteristics of magnetosphere ions, are mixed with variable magnetosheath ions. In the time interval 02:30 and 02:47 UT, the encountered mixtures is the well known low latitude boundary layer (LLBL) composition, which has a fully mixed ion energy structure. Besides the LLBL, there are some irregular mixtures, where some sporadic MS composition is observed in a stable background of the magnetospheric ion composition. TC-1 was skimming over the ellipsoidal MP at that time, hence these flux fluctuations should be regarded as local spatial structures at the MP instead of temporal variations due to the radial vibration of the MP. Otherwise, the background of the MS and magnetospheric ion composition should be alternately observed, in- stead of a stable background of the magnetospheric ion composition that was observed. Therefore, the fluctuated mixtures actually represent a transport of the MS ions across the MP. A possible ripple structures at the MP is shown in the schematic of Fig. 2.

Note that the duration of each fluctuation structure is much longer than the ion gyroperiod $(\sim 2 \mathrm{~s})$. For example, during 02:35-02:36 UT, some wavy flux tubes were recorded, and each tube persisted for $20 \mathrm{~s}$, and those fluctuations encountered later have even longer durations.

Figure 5 shows time-energy spectrogram of low energy electrons detected onboard the TC- 1 spacecraft. From top to bottom, are electron energy fluxes in the pitch angle range of $0-15^{\circ}, 75-90^{\circ}$ and $165-189^{\circ}$, respectively. The electron observations also show a mixture of MS and magnetospheric compositions. The higher energy population (lower flux) is the magnetospheric population, while the lower energy population (higher flux) is from the MS. 


$$
\text { Double Star(TC-1) }
$$

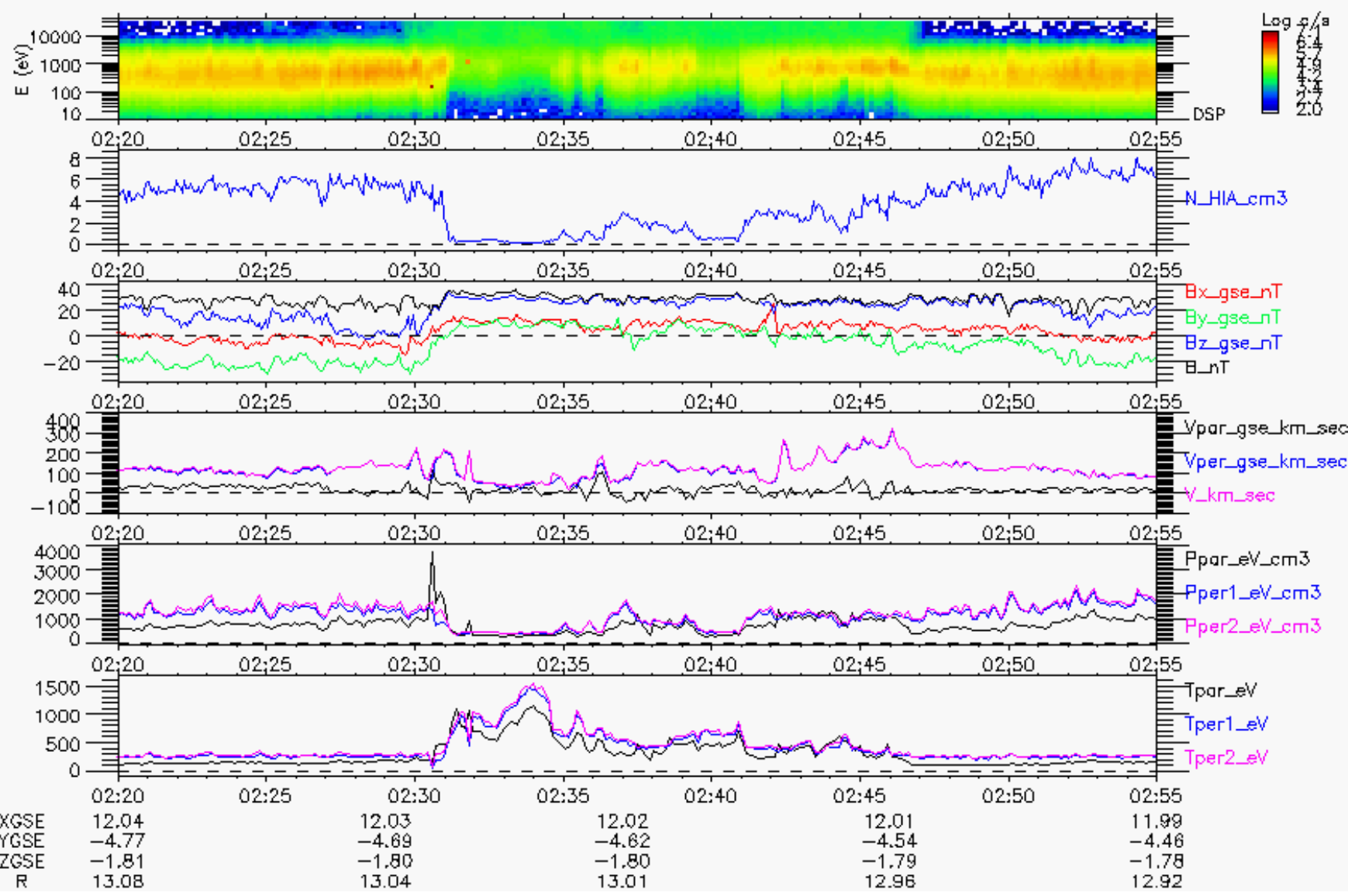

Fig. 4. Magnetic field and hot ion parameters from TC-1. From top to bottom, (a) hot ion time-energy spectrogram, (b) hot ion density, (c) components of the magnetic field, (d) ion bulk velocities. (e) and (f) components of hot ion pressure, and temperature, respectively.

\section{Discussion}

\subsection{Excursion velocity of the bow shock}

The small size of the Cluster tetrahedron allows the normal direction of the bow shock to be determined by a magnetic field gradient method (Shen et al., 2007). Then the velocity in the normal direction of the shock front can be estimated by the shock mass flux conservation equation, that is, $\rho_{u}\left(\boldsymbol{V}_{u}-V_{n} \boldsymbol{n}\right) \cdot \boldsymbol{n}=\rho_{d}\left(\boldsymbol{V}_{d}-V_{n} \boldsymbol{n}\right) \cdot \boldsymbol{n}$, where $\rho_{u}$ $\left(\rho_{d}\right)$ and $\boldsymbol{V}_{u}\left(\boldsymbol{V}_{d}\right)$ are the plasma density and velocity upstream (downstream) of the shock respectively, and $V_{n}$ is the excursion velocity in the normal direction (Schwartz, 1998). From the above equation, $V_{n}$ can be solved to be $V_{n}=\Delta(\rho \boldsymbol{V}) \cdot \boldsymbol{n} / \Delta \rho$. For the first bow shock encounter at 02:30 UT, the normal direction is determined to be $\mathbf{n}=(0.740,-0.580,-0.340)_{\mathrm{GSE}}$. The density and velocity upstream and downstream of the shock are $\rho_{u}=1.60 \mathrm{~cm}^{-3}$, $\boldsymbol{V}_{u}=(-453,18,-4)_{\mathrm{GSE}} \mathrm{km} / \mathrm{s}$ and $\rho_{d}=5.76 \mathrm{~cm}^{-3}, \boldsymbol{V}_{d}=(-92$, $-74,-112 \mathrm{k})_{\mathrm{GSE}} \mathrm{km} / \mathrm{s}$ respectively. Hence $V_{n}$ is evaluated to be roughly $156 \mathrm{~km} / \mathrm{s}$. Similarly, for the second bow shock encounter at $02: 47 \mathrm{UT}$, the normal direction is $\mathbf{n}=(0.744,0.141,-0.653)_{\mathrm{GSE}}$. The density and velocity upstream and downstream of the shock are $\rho_{u}=1.63 \mathrm{~cm}^{-3}$, $\boldsymbol{V}_{u}=(-437,13,20)_{\mathrm{GSE}} \mathrm{km} / \mathrm{s}$ and $\rho_{d}=7.16 \mathrm{~cm}^{-3}, \boldsymbol{V}_{d}=(-102$, $-31,-83)_{\mathrm{GSE}} \mathrm{km} / \mathrm{s}$, hence $V_{n}$ is approximately $65 \mathrm{~km} / \mathrm{s}$.

Which mechanism is responsible for the motion of the bow shock and MP is a long- standing issue. It has been proposed that certain plasma instabilities, for instance the convective Helmholtz-Kelvin instability, might be responsible for the motion of the magnetospheric boundary and that disturbances thus created propagate along the flanks of the magnetopause as a kind of magnetohydrodynamic surface waves (Anderson et al., 1968). An alternative approach is that periodic perturbations in the solar wind are responsible for driving the motion of the bow shock and magnetopause (Smit, 1968). Although here a large-amplitude excursion of MS was clearly observed, the driving source is not clear since neither an apparent correlation between the MS motion and the variance of solar wind parameters can be found nor large scale MHD waves were observed. The identification of the 

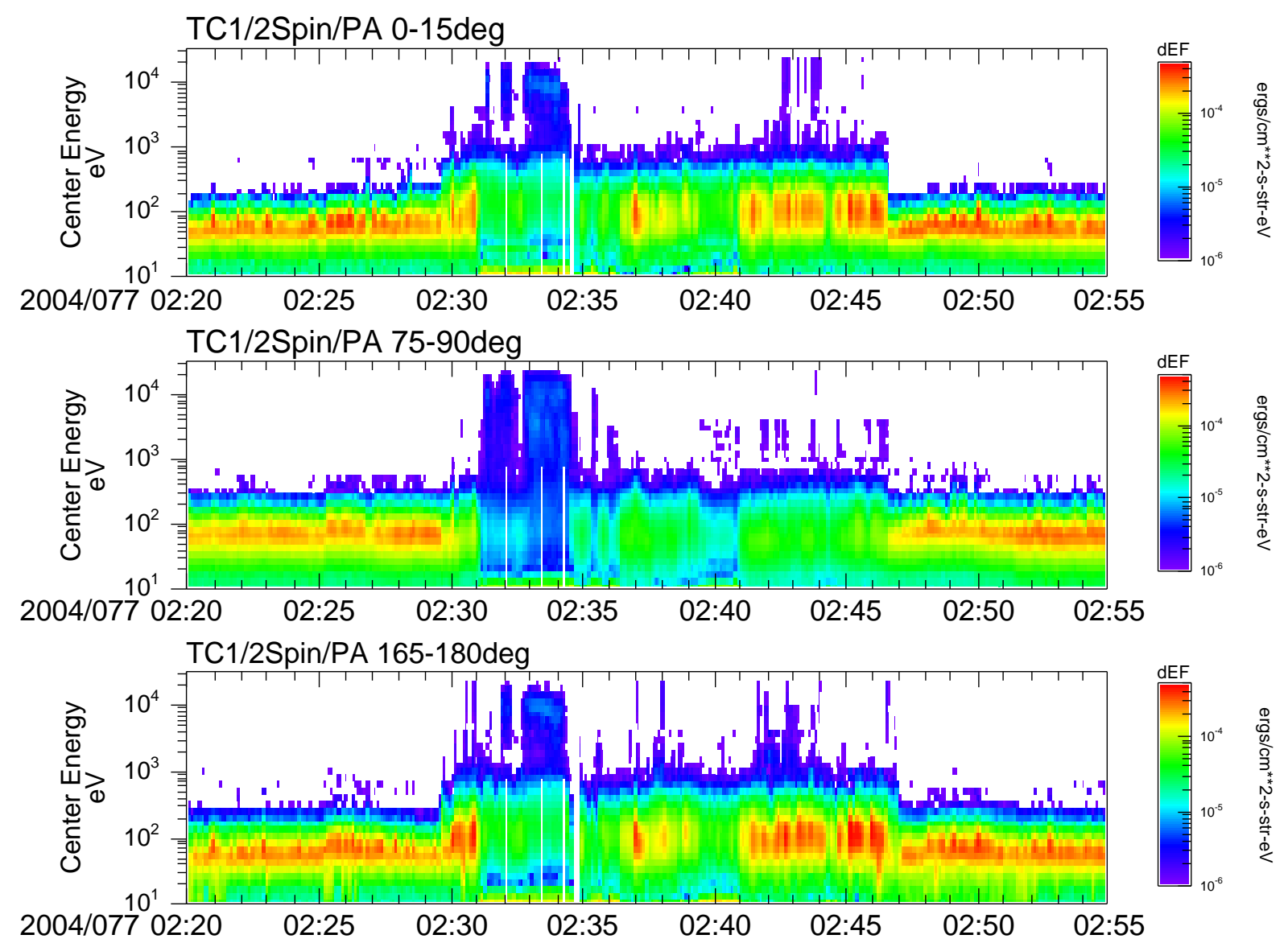

Fig. 5. Time-energy spectrogram of low energy electrons from TC-1. From top to bottom, are electron energy fluxes in the pitch angle range of $0-15^{\circ}, 75-90^{\circ}$ and $165-189^{\circ}$, respectively.

driving mechanism of the motion of bow shock and magnetopause is left for future investigation.

\subsection{Drift instability}

During the period TC-1 stayed at the MP, there were no apparent magnetic perturbation, hence an electrostatic type instability is responsible for the generation of the ripple structures (Fig. 2). Moreover, since there were prominent density gradient and the corresponding plasma pressure gradient at the MP, thus they can act as free energy to excite drift instability, whose fundamental physical picture is illustrated on the right side of Fig. 2. Here, a sinusoidal density perturbation is assumed. In the scenario of drift wave, the dynamics of electrons parallel to the magnetic field is adiabatic. Therefore, a plasma density perturbation directly connects to a plasma potential perturbation via the electron Boltzmann distribution in the parallel direction and an electric field in the plane perpendicular to the magnetic field builds up. Consequently, the $\boldsymbol{E} \times \boldsymbol{B}$ drift leads to propagation of the perturbation in the plasma diamagnetic drift direction, where the ion diamagnetic drift direction is eastward and opposite to that of electrons. In the MP environment, the electron perpendicular temperature is much lower than the ion perpendicular temperature (Phan et al., 2005). Thus, the electron diamagnetic drift velocity can be neglected and the drift wave propagates in the positive y direction with the ion diamagnetic drift velocity.

Drift waves are intrinsically three-dimensional and the plasma dynamics parallel to the magnetic field is a crucial parameter for stability. Due to an adiabatic and instantaneous electron response parallel to the magnetic field, the initial perturbation is linearly stable. However, when certain effects that lead to non-adiabaticity of electrons react, or in other words, the parallel dynamics of electrons is somewhat inhibited, the potential perturbation will increase slowly and the drift wave becomes unstable. Therefore, in the low frequency regime drift instability is essentially an electrostatic instability due to charge accumulation and accordingly electrostatic potential perturbation. This kind of manifestation in the present event is revealed in Fig. 6. The first panel shows the time-energy spectrogram of electrons in the energy range of $0.2-0.4 \mathrm{MeV}$ provided by the TC-1 HEED experiment between 02:34-02:44 UT (Cao et al., 2005). The second panel 


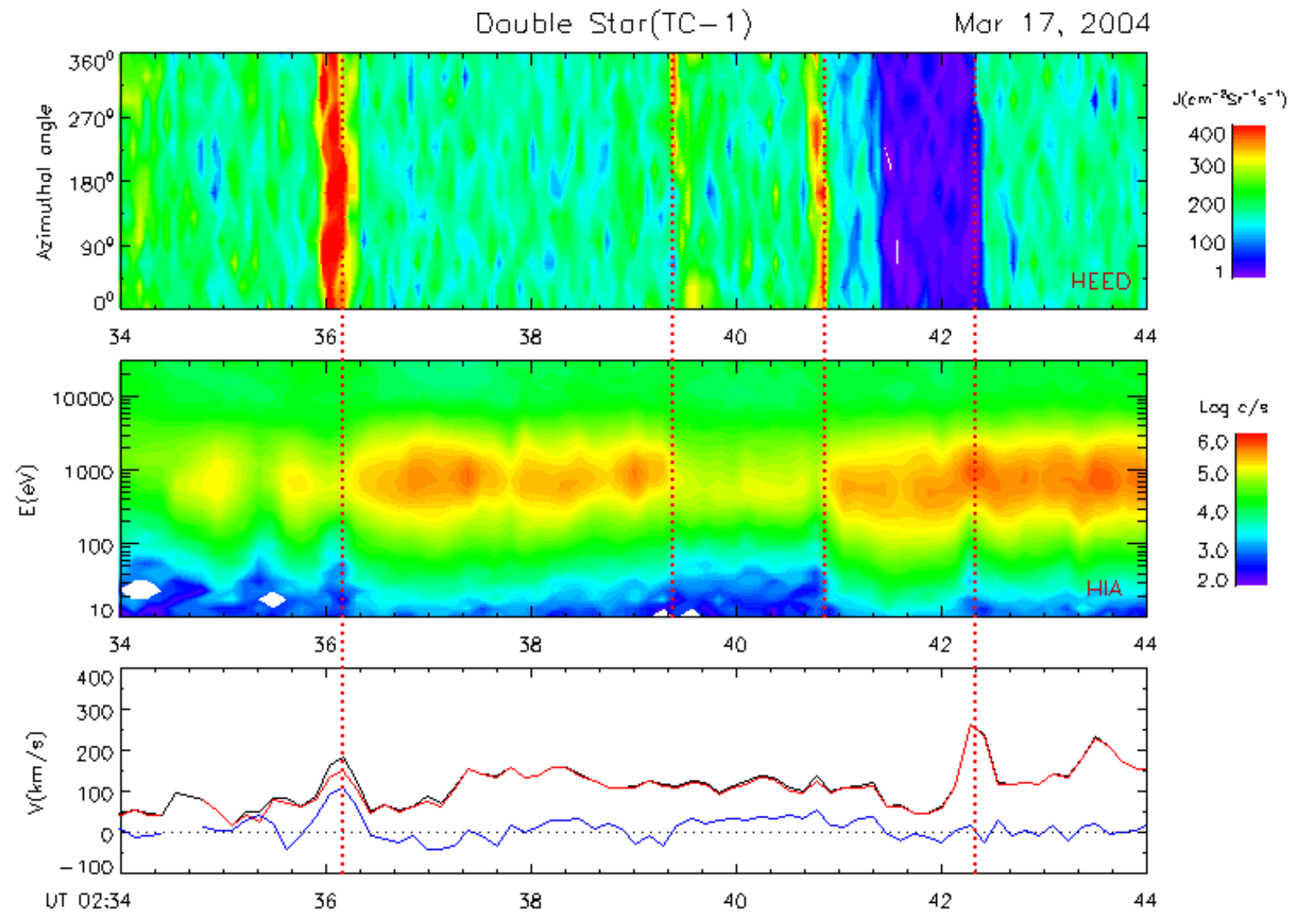

Fig. 6. (a) Time-energy spectrogram of electrons in the energy range of $0.2-0.4 \mathrm{MeV}$ recorded by the HEED experiment onboard the TC-1 spacecraft. The vertical axis is the azimuthal angle, which is defined in the plane perpendicular to the satellite spin axis. (b) HIA time-energy spectrogram. (c) Ion bulk velocities: the black curve represents the total velocity, and the blue and red represent parallel and perpendicular velocities, respectively.

is HIA time-energy spectrogram and the third panel shows the ion bulk velocities deduced from HIA measurements: the black curve represents the total velocity, and the blue and red represent parallel and perpendicular velocities respectively. In the interval 02:35:58-02:36:12 UT, a filament of electron flux up to $400 \mathrm{~cm}^{-2} \mathrm{sr}^{-1} \mathrm{~s}^{-1}$ was observed, which implies that a region of negative charge accumulation and accordingly a potential minimum is encountered. While during the period of 02:41:26-02:42:25 UT, a flux tube with ion flux below ten $\mathrm{cm}^{-2} \mathrm{sr}^{-1} \mathrm{~s}^{-1}$ was recorded, which implies that a region of positive charge accumulation and accordingly a potential maximum. Also, as shown by two short dashed lines, during the period of 02:39:22-02:40:51 UT, a minor potential minimum is exhibited, which is bounded by two minor regions of negative charge accumulation. Why the whole region of this potential perturbation did not display apparent variations of electron flux but only at the edges? Since electrons satisfy approximately the Boltzmann distribution and also the potential perturbation at that time is small, hence the electron perturbation is proportional to the ambient plasma density and inversely proportional to the plasma tempera- ture. Therefore, the electron perturbation and corresponding charge accumulation in the magnetospheric ambience are much smaller than that in the MS ambience and accordingly no apparent variations of electron flux are observed.

In comparison with the second panel in Fig. 6, it can be found that all the potential perturbations basically have a good correlation to the ion density perturbations. As shown in the schematic Fig. 2, a potential minimum corresponds to a low density region, where only the magnetospheric composition were encountered; while a potential maximum corresponds to a high density region, where the MS composition were met and mixed with the magnetospheric composition. This fact is coincident to the intrinsic feature of drift instability, and the electrostatic potential perturbation tightly ties to the plasma density perturbation. At the beginning there is no phase shift between them; however the phase shift increases rather slowly due to charge accumulation and eventually reaches to a small but finite value. Here, the minor potential minimum observed in the interval 02:39:2202:40:51 UT is exactly a growing potential perturbation during the evolution of drift instability. In fact, this growing 
potential minimum and the fully enhanced potential maximum encountered later during 02:41:26-02:42:25 UT make up spatially a complete sinusoid-like perturbation propagating in the positive y direction on one hand, while they also record temporally the history of potential growth on the other hand.

As shown by two long dashed lines at 02:36:10 and 02:42:20 UT, respectively, in Fig. 6, at the end of the record of both two major potential perturbations, a pulse of the perpendicular velocity is observed. These pulses are due to the $\boldsymbol{E}$ cross $\boldsymbol{B}$ motion in the nonlinear regime. In the linear stage of drift instability, although the potential perturbation grows up slowly, however both the potential and density perturbations are harmonically oscillated and no net mass transport across the boundary takes place. When the potential perturbation is high enough, the $\boldsymbol{E} \times \boldsymbol{B}$ motion will give rise to convective instability, causing mass interchange between the high density region and the low density region. Consequentially, the exchanged MS ion fluxes are observed in the interval 02:36:20-02:37:40 UT and after 02:42:20 UT, respectively. It is worthy to point out that associated with the first perpendicular velocity pulse, there is also a parallel velocity pulse, which implies that at this stage, the ion parallel motion is relevant, or in other words, the ion acoustic wave plays a role in the system evolution. For the second perpendicular velocity pulse, no parallel velocity pulse occurs. However, an apparent corresponding magnetic perturbation can be found from the third panel in Fig. 4. It indicates that at that stage, the instability is not merely electrostastic but electromagnetic, namely the shear Alfvén wave involves in the whole dynamics.

In contrast to the measurements of high energy electrons that reveal some evidence on charge accumulation and potential perturbation, the observations of low energy electrons as shown in Fig. 5 display no similar indication. The reason is thought that high energy electrons show non-adiabaticity in the parallel motion due to their larger effective viscosity and are responsible for the charge accumulation. Another issue is the stabilization of magnetic shear effect on the drift instability, since in the present event the MS magnetic field is sheared to the terrestrial magnetic field with an angle about $45^{\circ}$. The answer is that due to the bulk excursion of MS, a localized region with a small magnetic shear or without shear developed. It can be found out from the third panel in Fig. 4. After 02:47 till 02:51 UT, the plasma measurements clearly indicate that the satellite was already drifting away from the MP and entered into the MS, while the magnetic field records were still similar to that of the terrestrial magnetic field.

Finally, the perpendicular wavelength can be estimated by the product of each duration time of the filament structure and the ion diamagnetic drift velocity. The ion diamagnetic drift velocity is $v_{i t h} . \rho_{i} / L_{n}$, where $v_{i t h}$ is the ion thermal velocity, $\rho_{i}$ is the ion gyroradius and $L_{n}^{-1}=-d n / n d x$ is the scale length of the density gradient. Usually, at the MP the scale length of density gradient is typically a few ion gyroradii (Winske, 1996). Here, we assume that the ratio of the characteristic length to the ion gyroradius is about five, so the ion diamagnetic drift velocity is one-fifth of the ion thermal velocity and is about $30 \mathrm{~km} / \mathrm{s}$. Then, the perpendicular wavelength of drift waves can be estimated to be roughly from $1200 \mathrm{~km}$ to $5400 \mathrm{~km}$. Since a drift wave has a much larger parallel wavelength than its perpendicular wavelength, the volume of each flux tube is large and hence eventual particle transport by exchange of flux tubes is actually considerable. Based on this interpretation, it is interesting to point out that in contrast to the most investigated drift instability at the MP in the low hybrid regime and the assumed stationary turbulent diffusion, the observations presented here reveal the drift instability frequency is much less the ion gyrofrequency and consequently large-scale convective interchange seems to be a more efficient transport mechanism at the MP during periods of northward IMF.

\section{Conclusion}

In summary, a large-amplitude excursion of the MS in quiet solar wind conditions during period of northward IMF is reported, which was observed simultaneously by the Cluster and TC-1 spacecraft. The good coherence between the bow shock motion and the MP motion is revealed and the excursion velocity of the bow shock motion is analyzed by a multisatellite data analysis technique. Furthermore, the relevant plasma transport phenomenon in the form of flux fluctuations below the ion gyrofrequency at the MP is shown and interpreted as a manifestation of the drift instability. It is indicated that even in the case of IMF having a large shear to the terrestrial magnetic fields, a localized region at the MP without shear can develop due to the bulk motion of the MS and hence this stabilization factor in the drift instability is absent. In addition, evidence of charge accumulation and electrostatic potential perturbation related the evolution of drift instability are exhibited from the measurements of high energy electrons. All these potential perturbations show a good correlation to the ion density perturbations. Moreover, the relevant rapid $\boldsymbol{E} \times \boldsymbol{B}$ motion in the nonlinear stage and the corresponding mass exchange are recorded. Finally, based on rough estimation of the scale of drift wave lengths, it is pointed out that in contrast to the most investigated drift instability at the MP in the low hybrid regime and the assumed stationary turbulent diffusion, the observations presented here reveal the drift instability to be much less than the ion gyrofrequency and consequently large-scale convective interchange seems to be a more efficient transport mechanism at the MP during a period of northward IMF.

Acknowledgements. This work is supported by the National Natural Science Foundation of China (NSFC) under Grant No. 40621003, 40523006 and the National Basic Research Program of China 2006CB806305 and the Specialized Research Fund for State Key 
Laboratories and the French National Space Agency (CNES). C.L.C acknowledges the French Embassy in China for his government scholarship.

Topical Editor I. A. Daglis thanks P. J. Kellogg and another anonymous referee for their help in evaluating this paper.

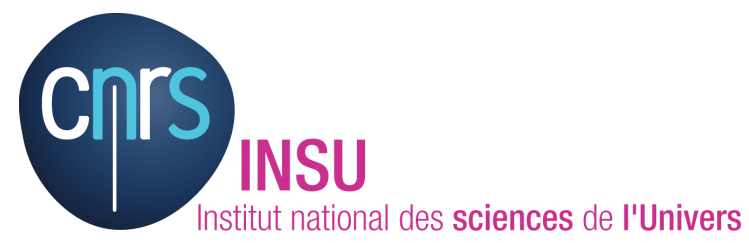

The publication of this article is financed by CNRS-INSU.

\section{References}

Anderson, K. A., Binsack, J. H., and Fairfield, D. H.: Evidence for hydromagnetic waves of 3- to 15-minute period on the magnetopause and their relation to bow shock spikes, J. Geophys. Res., 73, 2371-2386, 1968.

Balogh, A., Carr, C. M., Acuña, M. H., Dunlop, M. W., Beek, T. J., Brown, P., Fornacon, K.-H., Georgescu, E., Glassmeier, K.H., Harris, J., Musmann, G., Oddy, T., and Schwingenschuh, K.: The Cluster Magnetic Field Investigation: overview of in-flight performance and initial results, Ann. Geophys., 19, 1207-1217, 2001, http://www.ann-geophys.net/19/1207/2001/.

Berchem, J. and Russell, C. T.: The thickness of the magnetopause current layer, J. Geophys. Res., 87, 2108-2114, 1982.

Cao, J. B., Liu, Z. X., Yan, C. X., Cai, C. L., Li, Z. Y., Zhu, G. W., Wang, S. R., Zhao, H., Liang, J. B., Ren, Q. Y., Zai, Y. Y., Rème, H., Dandouras, I., Aoustin, C., Escoubet, P., Fazakerley, A., McKenna-Lawlor, S., and Dunlop, M.: First results of Chinese particle instruments in the Double Star Program, Ann. Geophys., 23, 2775-2784, 2005, http://www.ann-geophys.net/23/2775/2005/.

Gary, S. P. and Eastman, T. E.: The lower hybrid drift instability at the magnetopause, J. Geophys. Res., 84, 7378-7381, 1979.

Holzer, R. F., McLeod, M. G., and Smith, E. J.: Preliminary results from the OGO 1 search coil magnetometer: Boundary positions and magnetic noise spectra, J. Geophys. Res., 71, 1481-1486, 1966.

Horbury, T. S., Cargill, P. J., Lucek, E. A., Eastwood, J., Balogh, A., Dunlop, M. W., Fornacon, K.-H., and Georgescu, E.: Four spacecraft measurements of the quasiperpendicular terrestrial bow shock: Orientation and motion, J. Geophys. Res., 107, 1208, doi:10.1029/2001JA000273, 2002.
LaBelle, J. and Treumann, R. A.: Plasma waves at the dayside magnetopause, Space Sci. Rev., 47, 175-202, 1988.

Lemaire, J.: Impulsive penetration of filamentary plasma elements into the magnetospheres of the Earth and Jupiter, Planet. Space Sci., 26, 887-890, 1977.

Lemaire, J. and Roth, M.: Penetration of solar wind plasma elements into the magnetopause, J. Atrnos. Terr. Phgs., 40, 331335, 1978.

Kaufmann, R. L. and Konradi, A.: Explorer 12 magnetopause observations: Large-scale non-uniform motion, J. Geophys. Res., 74, 3609-3627, 1969.

Phan, T. D., Escoubet, C. P., Rezeau, L., Treumann, R. A., Vaivads, A., Paschmann, G., Fuselier, S. A., Attie, D., Rogers, B., and Sonnerup, B. U. O.: Magnetopause process, Space Sci. Rev., 118, 367-424, 2005.

Rème, H., Aoustin, C., Bosqued, J. M., et al.: First multispacecraft ion measurements in and near the Earths magnetosphere with the identical Cluster ion spectrometry (CIS) experiment, Ann. Geophys., 19, 1303-1354, 2001, http://www.ann-geophys.net/19/1303/2001/.

Rème, H., Dandouras, I., Aoustin, C., Bosqued, J. M., Sauvaud, J. A., Vallat, C., Escoubet, P., Cao, J. B., Shi, J., BavassanoCattaneo, M. B., Parks, G. K., Carlson, C. W., Pu, Z., Klecker, B., Moebius, E., Kistler, L., Korth, A., Lundin, R., and the HIA team: The HIA instrument on board the Tan Ce 1 Double Star near-equatorial spacecraft and its first results, Ann. Geophys., 23, 2757-2774, 2005, http://www.ann-geophys.net/23/2757/2005/.

Schwartz, S. J.: Shock and Discontinuity Normals, Mach Numbers, and Related Parameters, in: Analysis Methods for MultiSpacecraft Data, edited by: Paschmann, G. and Daly, P., 1998.

Shen, C., Dunlop, M., Li, X., Liu, Z. X., Balogh, A., Zhang, T. L., Carr, C. M., Shi, Q. Q., and Chen, Z. Q.: New approach for determining the normal of the bow shock based on Cluster four-point magnetic field measurements, J. Geophys. Res., 112, A03201, doi:10.1029/2006JA011699, 2007.

Smit, G. R.: Oscillatory Motion of the Nose Region of the Magnetopause, J. Geophys. Res., 73, 4990-4993, 1968.

Treumann, R. A., LaBelle, J., and Pottelette, R.: Plasma diffusion at the magnetopause: The case of lower hybrid drift waves, J. Geophys. Res., 96, 16009-16013, 1991.

Winske, D. and Omidi, N.: Diffusion at the magnetopause: Hybrid simulations, J. Geophys. Res., 100, 11923-11933, 1995. 